\title{
Grossesse, allaitement et vaccin contre le SRAS-CoV-2 : cadre éthique pour la prise de décision partagée
}

\author{
Jonathan S. Zipursky MD, Rebecca A. Greenberg IA PhD, Cynthia Maxwell MD, Tali Bogler MD MSc santé \\ communautaire
}

- Citation : CMAJ 2021 March 1;193:E312-4. doi : 10.1503/cmaj.202833-f; diffusion hâtive le 27 janvier 2021

Voir la version anglaise de l'article ici : www.cmaj.ca/lookup/doi/10.1503/cmaj.202833

$\mathbf{E}$ n décembre 2020, le Comité consultatif national de l'immunisation du Canada a déclaré que l'administration systématique du vaccin contre le coronavirus du syndrome respiratoire aigu sévère 2 (SRAS-CoV-2) aux personnes enceintes ou qui allaitent devrait être évitée jusqu'à ce qu'on dispose de suffisamment de données, mais que la vaccination de ces dernières pouvait être envisagée dans certaines situations où les avantages surpassent les risques ${ }^{1}$. Nous n'avons toutefois pas la même perspective du côté des États-Unis, où des associations de spécialistes ne déconseillent pas l'administration du vaccin contre le SRAS-CoV-2 à ces populations ${ }^{2,3}$. Nous croyons, d'un point de vue éthique, que le vaccin devrait être offert aux personnes enceintes, qui tentent de le devenir ou qui allaitent, et discutons de l'adoption d'une approche de prise de décision partagée par les fournisseurs de soins et ces dernières pour orienter leurs conversations.

Les principes fondamentaux de l'éthique médicale soutiennent que les décisions et interventions médicales doivent respecter l'autonomie de la personne, être équitables, être bénéfiques (bienfaisance) et ne pas causer de préjudices (nonmalfaisance). Empêcher les personnes enceintes ou qui allaitent d'être vaccinées contre le SRAS-CoV-2 limite leur autonomie et ne tient pas compte des facteurs individuels les concernant. Les personnes enceintes ont le droit de prendre des décisions qui servent leur intérêt supérieur, même si ce dernier va à l'encontre du bien-être du fœetus ${ }^{4}$. Leur exclusion catégorique de la vaccination ne leur permet pas de faire un choix en fonction de leurs valeurs et de leur situation personnelle. Même si les personnes enceintes jouissent de certains droits au Canada, des croyances normatives culturelles profondes voulant que ces dernières sacrifient leur propre santé pour assurer celle de leur enfant peuvent exister. Les principes d'équité en santé diraient plutôt que la santé de la mère est aussi importante que celle de son enfant ou de ses futurs enfants, et donc que toutes les per-

\section{POINTS CLÉS}

- Au Canada, on recommande d'éviter l'administration systématique du vaccin contre le coronavirus du syndrome respiratoire aigu sévère 2 (SRAS-CoV-2) aux personnes enceintes ou qui allaitent tant que les données seront insuffisantes; toutefois, d'un point de vue éthique, il est justifié d'offrir le vaccin à ces dernières, ainsi qu'à celles qui tentent de devenir enceintes.

- Les personnes enceintes ou qui allaitent ne courent pas toutes les mêmes risques, un fait qui devrait être pris en considération lors des discussions sur l'innocuité et les bienfaits de la vaccination.

- La prise de décision partagée peut aider les personnes enceintes ou qui allaitent à prendre une décision qui respecte leurs valeurs, et les fournisseurs de soins devraient appuyer la décision de celles-ci de se faire vacciner ou non.

- Les facteurs à prendre en considération dans les discussions sur la vaccination comprennent le degré de tolérance au risque, le risque individuel d'infection par le SRAS-CoV-2, celui de morbidité et de mortalité associées, les répercussions potentielles de la maladie sur le fœtus ou le nouveau-né, les responsabilités des membres de la famille et des proches aidants, de même que l'efficacité et l'innocuité du vaccin chez la personne enceinte et son fœtus.

sonnes, qu'elles soient enceintes ou non, devraient bénéficier d'un accès équitable au vaccin.

Les données d'essais cliniques actuellement disponibles ne prouvent pas que le vaccin contre le SRAS-CoV-2 est sûr pour les personnes enceintes ou qui allaitent, mais ne font pas non plus état de préjudices pour les mères et les fotus. Nous sommes d'avis que le refus de vacciner est justifié sur le plan éthique uniquement si des préjudices évidents, importants et imminents sont attendus. Si les vaccins vivants sont généralement déconseillés durant la grossesse en raison des risques théoriques 
d'infection fœtale, la plupart des autres types de vaccins sont considérés comme étant sûrs. Bien que la technologie utilisée dans les vaccins approuvés contre le SRAS-CoV-2 - l'ARNm soit nouvelle, les résultats préliminaires d'études de toxicité sur la reproduction chez les animaux ne montrent pas de préjudices pour les fœtus ${ }^{5}$. Par ailleurs, 23 femmes (12 dans le groupe recevant le vaccin et 11 dans le groupe sous placebo) sont devenues enceintes après avoir été vaccinées dans le cadre de l'essai clinique de Pfizer-BioNTech, et aucun effet indésirable n'a été signalé à ce jour ${ }^{6}$. En revanche, selon une étude en cours (revue systématique et analyse de données de surveillance recueillies par les Centers for Disease Control and Prevention des ÉtatsUnis), les femmes enceintes qui sont atteintes de la maladie à coronavirus 2019 (COVID-19) et qui présentent des symptômes courent un risque accru de maladie grave et d'issues indésirables de la grossesse, y compris d'accouchement prématuré7,8. Qui plus est, l'Academy of Breastfeeding Medicine a récemment publié une déclaration dans laquelle elle ne conseillait pas l'arrêt de l'allaitement après la vaccination contre le SRAS-CoV-2, les cellules $T$ et les anticorps produits à la suite de celle-ci pouvant se retrouver dans le lait maternel et protéger le nourrisson contre l'infection ${ }^{9}$. Le fait de prévenir les risques connus de la COVID-19, surtout lorsque les données limitées disponibles montrent que le vaccin pose peu de risques pour la santé, porte à croire que la vaccination est bénéfique pour la personne enceinte ou qui allaite et son enfant.

Il est également important de tenir compte de la probabilité qu'une personne soit infectée par le SRAS-CoV-2 et, le cas échéant, qu'elle développe une forme grave de la COVID-19. Au Canada, comme dans bien d'autres pays, les femmes sont surreprésentées dans les services de soins de première ligne et les services essentiels, ce qui accroît de manière disproportionnée leur risque d'infection ${ }^{10}$. De plus, certaines personnes voient leur risque augmenter en raison de problèmes de santé comme le diabète, l'obésité ou d'autres comorbidités ${ }^{7}$. Si une personne court un risque élevé de maladie, il est encore plus important qu'elle soit protégée.

Au Canada, les personnes à risque accru d'infection (p. ex. : travailleurs de la santé) et celles qui sont les plus vulnérables aux effets du virus seront vaccinées en premier ${ }^{11}$. Cette décision repose en partie sur le concept de réciprocité : les personnes qui sont prêtes à faire face au danger devraient pouvoir être protégées. En outre, la protection de ces personnes contre le SRAS-CoV-2 profitera à la société - concept d'utilité - puisque nombre d'entre elles jouent un rôle essentiel en première ligne et possèdent des compétences uniques qui les rendent difficilement remplaçables. Empêcher la vaccination des personnes de cette catégorie parce qu'elles sont enceintes ou qu'elles allaitent, c'est faire preuve de peu d'égards pour les risques auxquels elles s'exposent en soignant les autres.

Tant que les données ne montrent pas que les préjudices de la vaccination en surpassent les bienfaits, nous proposons d'offrir aux personnes enceintes ou qui allaitent la possibilité d'être vaccinées contre le SRAS-CoV-2. Dans le cadre d'une approche de prise de décision partagée, d'autres options devraient également leur être présentées, y compris celle d'attendre que plus de données soient disponibles avant de procéder à la vaccination et celle de ne pas se faire vacciner du tout et de simplement suivre les mesures de santé publique pour réduire le risque d'infection.

La prise de décision partagée est un processus au cours duquel un professionnel de la santé et un patient prennent, ensemble, des décisions médicales éclairées fondées sur des valeurs $^{12}$. Chaque personne enceinte ou qui allaite a ses propres facteurs de risque et considérations personnelles. La prise de décision partagée maximise l'autonomie de la personne et lui permet de prendre une décision qui respecte ses valeurs. À noter qu'une personne doit toujours se sentir soutenue dans la prise d'une décision relative à sa santé; par conséquent, nous recommandons l'utilisation d'un cadre qui facilite la prise de décision partagée en permettant à la personne concernée d'évaluer les risques et les avantages de chaque option, en fonction des données disponibles, ainsi que de ses valeurs et de sa situation. La personne fera appel à ses valeurs et à ses objectifs pour orienter la prise de décision et pourra, avec l'aide de son fournisseur de soins, choisir la solution qui est dans son intérêt supérieur. Certains des facteurs à prendre en considération dans les discussions sont présentés à l'annexe 1 (accessible en anglais au www. cmaj.ca/lookup/doi/10.1503/cmaj.202833/tab-related-content); on y mentionne notamment le degré de tolérance au risque, le risque individuel d'infection par le SRAS-CoV-2, celui de morbidité et de mortalité associées, les répercussions potentielles de la COVID-19 sur le fœtus ou le nouveau-né, les responsabilités des membres de la famille et des proches aidants, ainsi que l'efficacité et l'innocuité du vaccin chez la personne enceinte et son fœtus. Il est également important de tenir compte du degré de confiance qu'a la personne ou la communauté à l'endroit du système de santé. Certains groupes, dont les Noirs et les Autochtones, peuvent hésiter à se faire vacciner en raison du racisme et de l'abus de longue date ${ }^{13}$.

Les discussions sur l'innocuité du vaccin chez les personnes enceintes ou qui allaitent changeront au fur et à mesure que de nouvelles données seront publiées. Dans certaines régions, ces personnes peuvent s'inscrire à un registre après avoir été vaccinées, et on s'attend à ce qu'elles soient admissibles à des essais cliniques au début de 2021. Entre-temps, l'adoption d'une stratégie permissive est équitable, permettant aux personnes enceintes, qui tentent de le devenir ou qui allaitent de se faire vacciner. Cette stratégie peut être facilitée par la prise de décision partagée avec les fournisseurs de soins, qui va dans le sens des recommandations récemment formulées par la Société des obstétriciens et gynécologues du Canada ${ }^{6}$.

\section{Références}

1. National Advisory Committee on Immunization. Recommendations on the use of COVID-19 vaccines. Ottawa: Government of Canada; 2021. Accessible ici : www.canada.ca/en/public-health/services/immunization/national-advisory -committee-on-immunization-naci/recommendations-use-covid-19-vaccines. html (consulté le 11 janv. 2021).

2. Riley LE, Beigi R, Jamieson DJ, et al. Vaccinating pregnant and lactating patients against COVID-19. Washington (D.C.): American College of Obstetricians and Gynecologists; 2020. Accessible ici : www.acog.org/clinical/clinical-guidance/ practice-advisory/articles/2020/12/vaccinating-pregnant-and-lactating-patients -against-covid-19 (consulté le 14 déc. 2020). 
3. Society for Maternal-Fetal Medicine (SMFM) statement: SARS-CoV-2 vaccination in pregnancy. Washington (D.C.): Society for Maternal Fetal Medicine; 2020:1-3. Accessible ici : www.smfm.org/publications/339-society-for-maternal-fetal -medicine-smfm-statement-sars-cov-2-vaccination-in-pregnancy (consulté le 14 déc. 2020).

4. Criminal Code of Canada, R.S.C., 1985, c. C-46, s.223 (1)

5. Vaccines and Related Biological Products Advisory Committee meeting December 17, 2020. FDA briefing document: Moderna COVID-19 vaccine. Silver Spring (MD): United States Food and Drug Administration; 2020.

6. SOGC statement on COVID-19 vaccination in pregnancy. Ottawa: The Society of Obstetricians and Gynaecologists of Canada; 2020. Accessible ici : www.sogc. org/en/content/featured-news/SOGC_Statement_on_COVID-19_Vaccination _in_Pregnancy.aspx (consulté le 17 déc. 2020).

7. Allotey J, Stallings $\mathrm{E}$, Bonet $\mathrm{M}$, et al. Clinical manifestations, risk factors, and maternal and perinatal outcomes of coronavirus disease 2019 in pregnancy: living systematic review and meta-analysis. BMJ 2020;370:m3320. doi: 10.1136/bmj.m3320.
8. Zambrano LD, Ellington S, Strid P, et al. Update: characteristics of symptomatic women of reproductive age with laboratory-confirmed SARS-CoV-2 infection by pregnancy status - United States, January 22-October 3, 2020. MMWR Morb Mortal Wkly Rep 2020;69:1641-7.

9. Considerations for COVID-19 vaccination in lactation. Chicago: Academy of Breastfeeding Medicine; 2020. Accessible ici : www.bfmed.org/abm-statement -considerations-for-covid-19-vaccination-in-lactation (consulté le 17 déc. 2020).

10. Employment by class of worker, annual (x 1000): Table: 14-10-0027-01 (formerly CANSIM 282-0012). Ottawa: Statistics Canada. 2020. Accessible ici : www150. statcan.gc.ca/t1/tbl1/en/tv.action?pid=1410002701 (consulté le 17 déc. 2020).

11. Ismail SJ, Zhao L, Tunis MC, et al. Key populations for early COVID-19 immunization: preliminary guidance for policy. CMAJ 2020;192:E1620-32.

12. Grad R, Legare F, Bell NR, et al. Shared decision making in preventive health care What it is; what it is not. Can Fam Physician 2017;63:682-4.

13. Vohra-Miller S, Bhayana A. What good are COVID-19 vaccines if people are afraid? We need to build trust with racialized communities, specifically PSWs facing vaccine hesitancy. Toronto Star 2021 Jan. 8

\section{Intérêts concurrents : Aucun déclaré.}

Cet article a été révisé par des pairs.

Affiliations : Département de médecine (Zipursky), Université de Toronto; Division de pharmacologie et de toxicologie clinique (Zipursky), Centre des sciences de la santé Sunnybrook; Département de bioéthique (Greenberg), Système de santé Sinai, Département de pédiatrie, Université de Toronto; Département d'obstétrique et de gynécologie (Maxwell), Système de santé Sinai; Département de médecine familiale et communautaire (Bogler), Hôpital St. Michael, Toronto, Ont.

Collaborateurs : Tous les auteurs ont contribué à la conception du travail, ont rédigé le manuscrit et en ont révisé de façon critique le contenu intellectuel important; ils ont donné leur approbation finale pour la version destinée à être publiée et assument l'entière responsabilité de tous les aspects du travail.
Propriété intellectuelle du contenu : Il s'agit d'un article en libre accès distribué conformément aux modalités de la licence Creative Commons Attribution (CC BY-NC-ND 4.0), qui permet l'utilisation, la diffusion et la reproduction de tout médium à la condition que la publication originale soit adéquatement citée, que l'utilisation se fasse à des fins non commerciales (c.-à-d., recherche ou éducation) et qu'aucune modification ni adaptation n'y soit apportée. Voir : https://creativecommons.org/ licenses/by-nc-nd/4.0/deed.fr.

Remerciements : Les auteurs remercient sincèrement Nipa Chauhan, qui a conçu l'illustration, et Nathan Stall, qui a fourni des commentaires utiles sur les versions antérieures du manuscrit.

Déclaration d'intérêts : Tali Bogler est mariée à Nathan Stall, rédacteur associé pour le CMAJ, qui n'a pas participé au processus ayant mené au choix de cet article.

Correspondance : Jonathan Zipursky, Jonathan.Zipursky@sunnybrook.ca 